\title{
El cáncer de mama en México: evolución, panorama actual y retos de la sociedad civil
}

\author{
María Elena Maza-Fernández,(1) Elda Vecchi-Martini. ${ }^{(2)}$
}

\begin{abstract}
Maza-Fernández ME,Vecchi-Martini E. El cáncer de mama en México: evolución, panorama actual y retos de la sociedad civil. Salud Publica Mex 2009;5 I supl 2:S329-S334.
\end{abstract}

\section{Resumen}

Este artículo ilustra la evolución que han tenido las organizaciones de la sociedad civil de lucha contra el cáncer de mama, el papel que juegan, y los cambios que debe haber para que sus actividades impacten de fondo la calidad de vida de las mujeres y hombres con esta enfermedad. Hoy día, el concepto de sociedad civil se ha transformado y retoma cierta autonomía y penetra como sinónimo de participación. La sociedad civil es protagonista de temas centrales tales como salud, derechos humanos y asistencia social entre otros. Las asociaciones de cáncer de mama cuentan con libertad para organizarse, impulsar iniciativas a favor de los demás para mejorar su bienestar y desarrollar sus potencialidades en beneficio propio y de la comunidad en la que se desenvuelven. Estas asociaciones deben enfocarse en promover cambios en el sistema que resulten en una mejora de los servicios y en consecuencia de calidad de vida.

Palabras clave: sociedad civil; cáncer de mama; participación; iniciativas; calidad; servicio; México
Maza-Fernández ME,Vecchi-Martini E.

History, overview and challenges

of the breast cancer movement in Mexico.

Salud Publica Mex 2009;5 I suppl 2:S329-S334.

\section{Abstract}

This essay describes the history of the civil society breast cancer movement in Mexico, the role played by breast cancer NGOs and the changes they must undergo for their activities to impact the quality of life of men and women dealing with this disease. The concept of civil society today has been transformed, regaining a degree of autonomy and being at the center of a participatory democracy. Civil society takes a lead role in key issues such as health, civil rights, and public welfare. Breast cancer organizations have the liberty to organize and promote initiatives that will help others' welfare and develop their full potential for the benefit of themselves and their community. These organizations must focus on promoting changes in the system that will result in better services and better quality of life for their constituents.

Key words: civil society; breast cancer; movement; initiative; quality; welfare; Mexico
$\mathrm{M}$ éxico ha registrado cambios sustanciales en su estructura poblacional, situación que ha desembocado en una transición demográfica y epidemiológica. Ante el incremento de la esperanza de vida, ha surgido la necesidad de atender el envejecimiento de la población, acompañada de enfermedades crónicas y degenerativas.

En México, el cáncer de mama es la segunda causa de muerte por procesos tumorales en mujeres mayores de 25 años. ${ }^{1}$ Según estudios realizados por el Consejo

(I) American Cancer Society.

(2) Consultor independiente.

Fecha de recibido: 3 de noviembre de 2008 - Fecha de aprobado: 12 de diciembre de 2008 Solicitud de sobretiros: María Elena Maza Fernández. Carretera México-Toluca 5468 PH1, col. El Yaqui. 05320, México, DF. Correo electrónico:menmaza@msm.com,mmazacancer@prodigy.net.mx 
Nacional de Población (CONAPO), se calcula que para el año 2020 este grupo alcanzará los 38.8 millones, es decir, casi un tercio de la población total.

Ahora bien, debido al crecimiento poblacional de mujeres de 45 a 54 años, así como también del grupo de 35 a 44 años, la tasa de mortalidad por cáncer de mama en dicha población se incrementó durante la última década, de $13.06 \%$ en la década de 1990 a $14.49 \%$ en el año $2000 .^{2}$ Esto demuestra una tendencia ascendente a presentar cáncer de mama y morir por esta causa.

Infortunadamente, se ha observado que en la mayor parte de los casos, el diagnóstico de las pacientes se realiza en etapas muy avanzadas de la enfermedad (estadios III o IV). Este diagnóstico tardío implica que el tratamiento del cáncer es más costoso, más doloroso y con una posibilidad mucho más baja de curación. En otros casos, un mal diagnóstico anula la posibilidad de curación.

Ante esta situación, el gobierno federal estableció el "Programa de Acción para la Prevención y Control del Cáncer de Mamario, con base en el marco normativo vigente en materia de salud reproductiva y específicamente en el Proyecto de Norma Oficial Mexicana (ProyNOM-041-SSA2-2002)"* para la prevención, diagnóstico, tratamiento, control y vigilancia epidemiológica del cáncer de mama, publicado el 17 de septiembre del 2003 en el Diario Oficial de la Federación.

Con el diseño de esta NOM se pretende que la población esté mejor informada en relación con los riesgos del cáncer mamario y tome mejores decisiones para el autocuidado de su salud; de igual modo, debe mejorar la cobertura de detección y el seguimiento de casos, además de que es preciso diseñar y operar un sistema uniforme de información en el sector salud.

A partir del análisis de la situación actual y la capacidad de respuesta del Sistema Nacional de Salud, se definió el Modelo Operativo, ${ }^{3}$ el cual sintetiza dos tipos de estrategias. Por un lado, las medidas sustantivas que integran los elementos de coordinación intrasectorial e intersectorial, detección, diagnóstico, tratamiento, control de calidad, supervisión, evaluación e investigación $\mathrm{y}$, por otro lado, las medidas de apoyo que sirven para el fortalecimiento de la infraestructura.

En enero del año 2006, el gobierno federal anunció la cobertura universal del cáncer de mama a través del Seguro Popular. A pesar de esto, los servicios aún no llegan a las mujeres mexicanas en la mayor parte de las

\footnotetext{
* Esta norma se complementa con las siguientes: NOM-007-SSA21993, NOM-158-SSA1-1996, NOM-168-SSA1-1998 y NOM-017SSA2-1994.
}

entidades rurales. Los diagnósticos son todavía tardíos y los pronósticos desfavorables.

Pese a todos los esfuerzos realizados por el sector público, los recursos dispuestos hoy para educación, prevención, detección oportuna, tratamiento y cuidados paliativos en cáncer de mama son insuficientes. Es por ello que en los últimos 30 años han surgido organizaciones de la sociedad civil que buscan llenar los vacíos y procurar una mejor calidad de vida a los pacientes con cáncer en México.

En las últimas dos décadas, la sociedad civil ha cobrado una notable importancia en México. Su presencia y participación se han convertido en una pieza clave en diversas áreas, como salud, educación y desarrollo rural. Más aún, ha participado en temas que se consideran monopolio del mercado o el Estado, como la integración comercial y la seguridad pública.

La nueva relación gobierno-sociedad muestra una mayor participación social que tiende a aumentar. En realidad, el concepto "sociedad civil" sustituyó de manera paulatina al concepto "pueblo" y se le confirió una cierta autonomía y una no lucha por el poder.

La tarea fundamental de una organización civil consiste en movilizar recursos locales, públicos y privados para aplicarlos con fines sociales. Se persigue la inclusión de los diversos actores, pero con independencia de los gobiernos o programas gubernamentales, aun cuando se prevean oportunidades de colaboración con ellos.

El rápido crecimiento de fundaciones en el plano mundial ha conducido a la creación de organizaciones, redes y grupos que reconocen el importante papel que juegan las organizaciones civiles en el desarrollo local. Éstos buscan ofrecer espacios de vinculación, fortalecimiento mutuo y sobre todo contribuir al desarrollo social viable en la realidad mexicana.

La sociedad civil es capaz de detectar de forma objetiva la problemática y promover la corresponsabilidad ciudadana, solidaria y subsidiaria, en la solución de los problemas sociales de la localidad; la finalidad es que los donantes o inversionistas sociales se sumen a través de las donaciones que realizan. Ofrece información, seguimiento y evaluación de los proyectos de inversión social y canaliza de modo profesional y eficiente recursos para el desarrollo local. Además, vincula actores, sectores e iniciativas y crea sinergias en beneficio de la comunidad.

En su origen, el concepto de sociedad civil en México fue marginal y netamente teórico. A partir del terremoto de 1985, su conceptualización se intensifica, extiende y penetra como sinónimo de participación, de la no manipulación partidaria y gubernamental, y la lucha por derechos por parte de la sociedad. Sin duda, se trató de una vía de expresión contra la cultura del cor- 
porativismo y el clientelismo político, hasta convertirse en un concepto aliado de "ciudadanía" o "democracia participativa", que se consolida en el lenguaje sociológico ideológico y político en 1988.

No debe olvidarse tampoco que el concepto de sociedad civil en México surge como concepto liberador contra las formas corporativas establecidas por el régimen de partido de Estado y, al mismo tiempo, como instrumento que favorece los cambios neoliberales del Estado mexicano y el de su papel en la economía.

Se puede considerar como antecedente remoto de las actuales organizaciones no gubernamentales (ONG) y organizaciones civiles el periodo instaurado por la dominación española. Durante la Conquista y la Colonia, a pesar del poderoso aparato gubernamental, se crearon instituciones que operaron con relativa independencia de los poderes gubernamentales. Más tarde, en los años posteriores a la Independencia, en la confrontación entre liberales y conservadores durante el Porfirismo y más adelante en la época correspondiente a la Revolución Mexicana, ya se advierte un mayor número de acciones e instituciones que pueden considerarse antecedentes de las actuales ONG y organizaciones civiles; pueden mencionarse la celebración de Semanas Sociales, la fundación de las Cajas de Ahorro Rurales (Método Raiffeisein), la fundación del Partido Católico Nacional, la Conferencia Nacional de Círculos Obreros, etc., entre 1910 y 1912.4

En estos mismos años también aparecen la fundación de la Escuela Libre de Derecho, la Gran Dieta de Zamora en 1913, la fundación de la Confederación Nacional Católica del Trabajo (CNCT) y el Secretariado Social Mexicano (SSM) en 1923. En ese mismo año, con la conquista de su autonomía, se funda la Universidad Nacional Autónoma de México (UNAM) y la campaña por la candidatura presidencial de José Vasconcelos que despertó la conciencia cívica de un importante sector de la sociedad mexicana. Este hecho se repitió con la candidatura presidencial del Gral. Juan Andrew Almazán en 1939, si bien las manipulaciones electorales de ambos procesos dejaron por largos años un sentido de frustración y apatía cívica y política en México. ${ }^{4}$

En 1951 se constituyó el Frente Mexicano Pro Derechos Humanos (FMPDH), pionero de todos los posteriores, el Movimiento Familiar Cristiano (MFC), fundado en 1958, como obra filial y autónoma de la ACM. Ese mismo año se creó el Centro Nacional de Ayuda de las Misiones Indígenas (CENAMI), antecedente de los numerosos organismos actuales de promoción indígena, como la Fundación Mexicana de Desarrollo Rural (FNDR).

En el decenio de 1970 se puede observar una expansión de las ONG -construcción de ciudadanía y solidaridad- que se pueden clasificar en dos clases de asociaciones: las de promoción y defensa de derechos y aquéllas prestadoras de servicios. Estas últimas buscan ser concesionarias para la operación de programas y servicios públicos y realizar acciones de bienestar y desarrollo. Este concepto de fundaciones combina asociacionismo, filantropía, asistencialismo y voluntariado. ${ }^{5}$ Nace en estos años la Asociación Mexicana de Lucha contra el cáncer "AMLCC".

Es necesario señalar que el peso del sismo de 1985 y el debilitamiento del régimen político promovieron el desarrollo comunitario al trabajar en la problemática de salud o la defensa de los derechos humanos. Estos fenómenos sentaron las bases para la creación de nuevos modelos de acción colectiva. En estos años se constituyó el Grupo de Recuperación Total Reto A.C.

A este respecto, el informe "Invertir en Salud", elaborado en la década de 1990 por el Banco Mundial, tuvo gran efecto en la atención prestada a la salud, en particular la labor de las ONG. En dicho documento se propone la conveniencia de encargarles algunas tareas gubernamentales y ofrecerles apoyo financiero, bajo el argumento de ser éstas más eficientes que las instituciones públicas. Asimismo, es necesario recordar que el nivel de pobreza se acentuaba y los programas sociales escaseaban. Es en esta década y la siguiente cuando surgen nuevas organizaciones, entre ellas la Fundación Cimab, FUCAM e Instituto Carso para la Salud, que promueven los derechos humanos y de los pacientes, así como la investigación, y se impulsan dentro de la asistencia oficial del desarrollo.

Las ONG en México son sobre todo fundaciones donantes, organizaciones independientes, autónomas, privadas y sin fines de lucro, dedicadas a atender las necesidades críticas de la comunidad y elevar la calidad de vida. En México se han establecido bajo diferentes condiciones; ${ }^{*}$ algunas han nacido con apoyos internacionales, otras se han conformado por ciudadanos que han conjuntado esfuerzos y creado sinergias en beneficio de sus comunidades; otras han nacido por la iniciativa de empresarios comprometidos con el desarrollo social y algunas más han recibido apoyos de instancias gubernamentales. ${ }^{6}$

\footnotetext{
* A pesar de que son organizaciones de individuos que persiguen un objetivo, nacen bajo diferentes circunstancias. Tal es el caso de la asociación civil, que "es una organización de individuos que persigue un fin lícito y el cual no es preponderantemente económico ni tampoco especulativo, por lo tanto los asociados que la integran no buscan obtener utilidades económicas de las funciones que realiza la asociación". Este tipo de asociaciones se rige por los documentos denominados "estatutos sociales", los cuales deben realizarse por escrito e inscribirse en el Registro Público de la Propiedad y es necesario que se efectúen en una escritura pública,
} 
Por su parte, la sociedad civil, de acuerdo con el Código Civil para el Distrito Federal, artículo 25, es un contrato por el cual "los socios se obligan mutuamente a combinar sus recursos o sus esfuerzos para la realización de un fin común, de carácter preponderantemente económico, pero que no constituya una especulación comercial." Este tipo de sociedad goza de personalidad jurídica tal y como lo establece el artículo 25, fracción III del Código Civil para el Distrito Federal, por lo que puede obligarse a celebrar contratos, comparecer a juicio, actuar en el ámbito jurídico, etc., y sujetarse a lo que dispongan sus estatutos sociales y a lo que sea necesario para el cumplimiento de su finalidad.

Si bien es cierto que el origen de las fundaciones marca una forma de operación específica, éstas conciben su trabajo como la creación de un espacio de vinculación de fuerzas de los diversos actores sociales, incluido el gobierno, para el desarrollo de proyectos específicos, formación y fortalecimiento de redes para la consecución del desarrollo comunitario.

Es indispensable que el gobierno federal reconozca la destacada labor de las organizaciones de la sociedad civil que trabajan activamente en la lucha contra el cáncer de mama en México, así como las acciones que han emprendido para generar mayor conciencia social sobre este problema de salud pública. Es crucial que las autoridades y las organizaciones sociales colaboren en equipo para enfrentar con éxito este trastorno.

En la actualidad, la Secretaría de Salud registra 76 organizaciones de la sociedad civil relacionadas con el cáncer en toda la República Mexicana. A pesar de esta proliferación, no existe hoy en día un movimiento civil organizado que luche por los derechos del paciente con cáncer ni por las guías de detección oportuna asentadas en la NOM.

En la actualidad, las organizaciones activas se reconocen sobre todo por sus programas asistenciales o bien por sus campañas de concientización sobre la enfermedad y los métodos de detección oportuna.

Las principales organizaciones de lucha contra el cáncer de mama en México son Grupo Reto, con presencia en las 32 entidades federativas; Fundación Cim*ab, con sedes en la Ciudad México y Monterrey; FUCAM, que en fechas recientes ha suscrito convenios con el INMEGEN y el Gobierno del Distrito Federal; Asociación Mexicana de Lucha Contra el Cáncer; y la iniciativa Tómatelo a Pecho de Funsalud e Instituto Carso para la Salud.

A continuación se describen de manera sinóptica cada una de las organizaciones arriba mencionadas.

\section{Grupo de Recuperación Total Reto, A.C.}

Por iniciativa de la Sra. Cecilia Vildósola de Sepúlveda, un grupo de ocho mujeres que habían pasado por la enfermedad crearon en abril de 1983 el Grupo de Recuperación Total (Reto) para dar apoyo a las mujeres con este diagnóstico. Se constituyó oficialmente como una asociación civil con fines no lucrativos el 27 de marzo de 1985.

En la actualidad, la oficina de la Ciudad de México está integrada por 80 voluntarias, casi todas mujeres operadas de cáncer mamario, las cuales prestan sus servicios de forma desinteresada. Su misión consiste en promover la salud integral en el área del cáncer mamario. Esto significa suministrar apoyo a través de sesiones para lograr la recuperación física y emocional de las pacientes y reintegrarlas a la sociedad. Al establecer el objetivo, su trabajo se ajustó al modelo del Grupo Reach to Recovery de la American Cancer Society. Esto consiste en que una voluntaria operada y recuperada visita a una mujer recién operada para platicar con ella, escucharla y llevarle una bolsa de recuperación. Bajo este esquema nace el programa de rehabilitación y cuenta con otros programas: Programa Educativo sobre Detección Oportuna de Cáncer de Mama, Programas de Ayuda Psicológica y Emocional al paciente, Programa de Cuidados Paliativos y Apoyo para Quimioterapias. De igual modo, se estableció un convenio con la UNAM para encontrar una fórmula para comenzar a elaborar prótesis mamarias y a partir de 1989 el Grupo Reto comenzó con la fabricación de estos dispositivos.

Hoy en día, Reto tiene presencia en todas las entidades federativas bajo una red de oficinas independientes y autónomas.

\section{Asociación Mexicana contra el Cáncer de Mama, A.C.}

La Asociación Mexicana contra el Cáncer de Mama, A.C., Fundación $\mathrm{Cim}^{*}$ ab, es una institución no gubernamental sin fines de lucro, surgida en octubre del 2002 por iniciativa de Alejandra de Cima Aldrete y Bertha Aguilar; su objetivo es difundir información actualizada que promueva la detección oportuna del cáncer de mama y con ello reducir el número de muertes.

La Fundación Cim*ab realiza su labor a través de una red de voluntarias y miembros en dos centros de información, uno en la Ciudad de México y otro en Monterrey. En dichos centros proporciona apoyo psicológico y emocional a las mujeres y familias afectadas. Informa 
a través de pláticas sobre la autoexploración mamaria y métodos de detección oportuna.

La Fundación Cim*ab se une con empresas para hacer grandes campañas educativas e informativas. Se difundió así la campaña "Favor de Tocar", la cual es muy sugerente y pretende fomentar la autoexploración. Presentó un cortometraje "Un día más" en el que 16 sobrevivientes de cáncer de mama ofrecen sus testimonios. Lleva a cabo hoy en día un programa en comunidades rurales con la colaboración de líderes de cada comunidad y expertos indigenistas. El gran reto de Fundación Cim*ab es crear conciencia sobre la enfermedad y difundir la idea de que el cáncer de mama no es sinónimo de muerte.

En el último año, Cim*ab se ha posicionado como la institución "hermana" de Susan G. Komen Foundation dentro de la Iniciativa Global Komen. A través de ésta se busca crear un movimiento global de lucha contra el cáncer de mama y buscar cambios favorables en las políticas públicas.

\section{Asociación Mexicana de Lucha contra el Cáncer "AMLCC"}

La institución se fundó en 1972 como una organización no gubernamental, sin fines de lucro, constituida con el propósito de colaborar en los programas preventivos contra el cáncer instituidos por la Secretaría de Salud en centros hospitalarios de todo el país.

Se considera una institución de segundo nivel, a través de la cual se reciben donativos y se canaliza a otras instituciones.

La tarea principal es equipar con tecnología de punta a todos los hospitales e instituciones pertenecientes a la Secretaría de Salud y atender a pacientes de escasos recursos que no cuentan con seguridad social. Las trabajadoras sociales dentro de los hospitales son las encargadas de realizar un estudio socioeconómico de los pacientes para definir el nivel de apoyo que necesitan. La parte del tratamiento que no puede cubrirse se canaliza a otras instituciones que atienden este tipo de enfermedades.

Con el diseño y la creación de las famosas alcancías se inició el proyecto de recaudación de fondos. A pesar de que es aún el pilar de la asociación, el organismo necesita trabajar en otras áreas para la recaudación de fondos.

Gracias a las alianzas con Avon y otras ONG, se han instalado equipos para la detección de cáncer de mama en toda la República Mexicana, se han comprado unidades móviles e instalado clínicas para atender a mujeres que no tienen acceso a los servicios de salud y que viven en zonas apartadas. En estas clínicas también se realizan estudios de Papanicolaou.
Hoy en día, uno de sus grandes proyectos es el de trabajar con todos los niños y adolescentes en el ámbito educativo en materia de prevención con pláticas sobre buenos hábitos y detección oportuna a través del programa "Hoy por mis padres, mañana por mí".

AMLCC pretende formar nuevas asociaciones estatales, con el fin de cubrir toda la República Mexicana y, por lo tanto, se ha invitado a diferentes organismos para unirse a AMLCC.

\section{Fundación Mexicana de Fomento Educativo para la Prevención y Detección Oportuna del Cáncer de Mama}

Nace por iniciativa del Dr. Fernando Guisa Hohenstain con la misión de hacer del cáncer mamario una enfermedad del pasado. Establece el primer instituto de enfermedades de la mama en América Latina con la finalidad de proporcionar un apoyo integral a las pacientes. Se ha enfocado en los programas de mastografías para todas. Por esta razón, se firmó un convenio para realizar dichos estudios de forma gratuita en las zonas marginadas del DF. Este programa ha causado controversia en el gremio por la calidad de los servicios que ofrecen, pero no ha dejado de contar con el apoyo del gobierno federal. En fecha reciente, FUCAM suscribió un convenio con el Instituto Nacional de Medicina Genómica (INMEGEN) cuyo propósito es que la fundación proporcione al instituto las muestras para realizar los estudios genómicos del cáncer de mama en la población mexicana.

\section{Tómatelo a Pecho}

Es un proyecto difundido por el Instituto Carso de la Salud en el año 2008 en colaboración con la Fundación Mexicana para la Salud. Es la primera iniciativa en México que aborda de manera integral el programa de cáncer de mama en México. "Tómatelo a Pecho" combate el problema en cuatro líneas de acción: información (revisión sistematizada en políticas públicas y análisis de prospectivas y fortalecimiento de la comunidad educativa); investigación en colaboración con el INMEGEN y el INSP; instituciones fortalecidas a través de apoyos; e innovación.

\section{Conclusiones}

Debe reconocerse que la multiplicación de las ONG constituyó una expresión de un proceso de autoestructuración de la sociedad civil en una economía de lucha por derechos y libertades civiles y ha dado lugar a acciones del Estado que permiten la participación, la integración social y la unión para actuar frente a las injusticias y 
desigualdades; ha quedado en el olvido lo que alguna vez dijo el Virrey Marqués de Croix: “...De una vez y por lo venidero, deben saber los súbditos...que nacieron para callar y obedecer y no para discutir y opinar en los altos asuntos de gobierno", según lo consigna la Historia minima de México de Daniel Cosío Villegas.

Las organizaciones de la sociedad civil de lucha contra el cáncer de mama en México han tenido hasta la fecha un papel esencialmente asistencialista y educativo. No es sino hasta la última década que las ONG empiezan a reconocer su función de representantes de la sociedad civil y comienzan a luchar por los derechos de los pacientes y tratar de tener un efecto en las políticas públicas.

Con la inclusión del cáncer de mama en el fondo de enfermedades catastróficas del Seguro Popular, cada día los apoyos para medicamentos se vuelven menos necesarios, no así los grupos de apoyo y, lo que es más importante aún, la vigilancia para que las pacientes reciban atención pronta y de calidad.

Según el ejemplo de los grupos de VIH-SIDA, las organizaciones de lucha contra el cáncer de mama empiezan a buscar mejoras en los servicios otorgados por el sistema de seguridad social mexicano.

A pesar de estos esfuerzos aislados, todavía no se observa una cohesión entre los grupos que pueda presentar un frente común ante las autoridades y así generar cambios de fondo en la manera en que hoy se enfrenta este padecimiento. Por otro lado, es importante resaltar que casi todas estas organizaciones son grupos de pacientes que no han logrado un nivel de profesionalización que los lleve a unificar criterios de detección oportuna basados en evidencia. Mientras no se consiga que las demandas presentadas tengan el respaldo de evidencia y una coalición representativa de la sociedad, es difícil esperar cambios sustanciales en favor de la mujer mexicana en su lucha contra esta enfermedad.

\section{Referencias}

I. Programa de Acción Cáncer de Mama 200I-2006, p. 17

2. Fuente de la INEGI - CONAPO.

3. Programa de Acción Cáncer de Mama 2001-2006, p. 31.

4. Álvarez Icaza J, CENCOS. El papel de las organizaciones civiles en la promoción del desarrollo rural en México. México: Editorial ERA, 1996:I5. 5. Graciela Biagini, UBA-UNLU,Argentina Sociedad Civil y salud en Latinoamérica: aproximaciones al estado del arte de las investigaciones. Disponible en: www.lasociedadcivil.org/uploads/ciberteca/g-biagini.pdf. 6. Centro Mexicano para la Filantropía. Historia de las fundaciones comunitarias en México. 